\title{
Smartphone GP consultation app cost CCG $£ 150000$ in first two months
}

Clarification - The CCG has pointed out that the surgery referred to in this story (BMJ 2018;360:k221, doi:10.1136/bmj.k221) was previously known as The Lillie Road Medical Centre (full address is 139 Lillie Road Health Centre) and is now called the
GP at Hand practice. The CCG also made clear that The Lillie Road Medical Centre/GP at Hand practice is in no way not affiliated to The Lillie Road Surgery, which is a separate surgery based in Fulham, West London. 